\title{
Prosthetic Management of a Maxillary Alveolar Defect with an Implant- Retained Fixed Prosthesis: A Clinical Report
}

\author{
Yazıcıoğlu $Y^{1}$, Özkurt-Kayahan $Z^{1 *}$, Özçakır-Tomruk $C^{2}$ and Katipoğlu $B^{3}$ \\ ${ }^{1}$ Faculty of Dentistry, Department of Prosthodontics, Yeditepe University, Istanbul, Turkey \\ ${ }^{2}$ Faculty of Dentistry, Department of Oral and Maxillofacial Surgery, Yeditepe University, Istanbul, Turkey \\ ${ }^{3}$ Faculty of Dentistry, Department of Oral and Maxillofacial Surgery, Istanbul University, Istanbul, Turkey
}

\begin{abstract}
Dentoalveolar defects resulting in the loss of alveolar ridge and attached mucosa is generally a challenge for the clinician to restore the defect area. The aim of this clinical report is to describe the prosthetic management of a maxillary alveolar defect. Treatment included 4 implants with a maxillary implant-retained fixed prosthesis. The esthetic and functional requirements of the patient were fulfilled.
\end{abstract}

Keywords: Implant; Rehabilitation; Dentoalveolar defects; Fixed prosthesis

\section{Introduction}

Dentoalveolar defects caused by maxillofacial trauma, car accidents, gunshot injuries, removal of tumors, periodontal diseases and/or traumatic tooth extractions often results in the extensive loss of alveolar bone, tooth and gingival tissues, causing esthetic and functional difficulties $[1,2]$. These cases have always been the most challenging situations in restorative procedures, because the dentoalveolar defects are likely to reduce the retention and stability of prosthesis $[3,4]$. There are different treatment modalities to replace missing teeth and soft and hard tissues, such as removable partial dentures, fixed partial dentures or implant-retained prosthesis [5].

The removable partial dentures are easy to maintain the oral hygiene and provide lip support in large dentoalveolar defects with lower cost and shorter treatment time [1]. Nevertheless, the removable prosthesis may not be accepted by patients because of the esthetic impairments and discomfort related to their base extension [6]. Conventional tooth supported fixed partial dentures have long been regarded as the standard of care in replacement of single and multiple missing teeth $[7,8]$. This may be an ideal treatment option where implants are contraindicated or patients refuse the implant therapy. However, natural abutment teeth with sufficient bone support are required for these kinds of prosthesis. Dental implants are a compatible, successful and predictable treatment modality, offering enhanced esthetics and biomechanical advantages. The increase in their clinical applications has determined a considerable reduction in the use of removable prosthesis, although complicated surgical procedures may be needed especially in large defect areas, such as augmentation, distraction or sinus lift for implant placement [1,9].

This article describes the prosthetic management of a maxillary dentoalveolar defect caused by removal of a dentigerous cyst in a young female patient. The defect area was restored with implant-supported metal-ceramic fixed partial denture with gingival-colored porcelain.

\section{Case Report}

A 29-year-old female patient was referred to the Department of Prosthodontics, Istanbul University, and Faculty of Dentistry for dental rehabilitation. The patient's history revealed that she had a dentigerous cyst defect. She had been wearing a removable partial denture with precision attachment for 6 years and complained about the insufficient esthetic, function and comfort (Figure 1). Intraoral examination revealed the lack of the maxillary anterior residual ridge on the right side, with the loss of maxillary right central and lateral

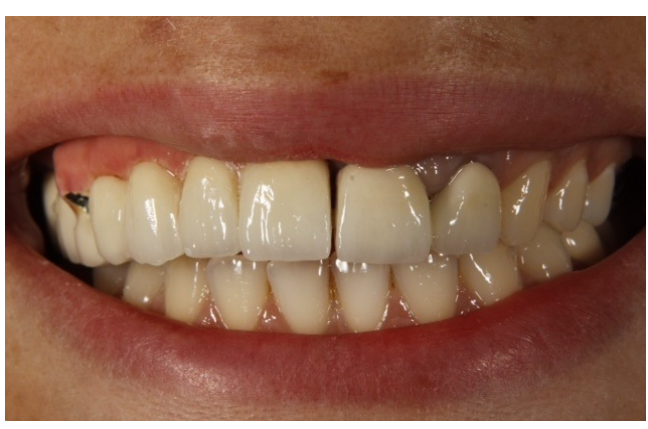

Figure 1: Existing denture of the patient.

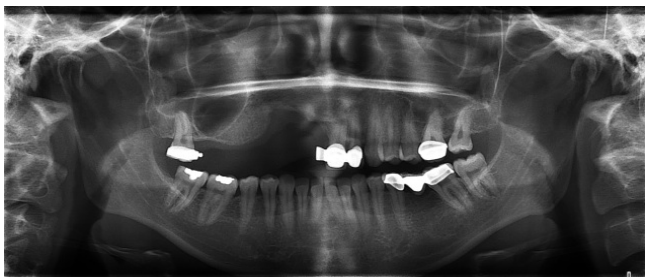

Figure 2: Initial panoramic radiograph.

incisors, canine, first and second premolar teeth, first and second molar teeth. The maxillary left central and lateral incisors had also metalceramic crowns (Figure 2). The patient had an Angle Class I occlusion, the temporomandibular joints were asymptomatic and jaw movement was in normal limits. The patient was reluctant for another removable

${ }^{*}$ Corresponding author: Zeynep Özkurt-Kayahan, Associate Professor, Faculty of Dentistry, Department of Prosthodontics, Yeditepe University, Istanbul, Turkey, Bağdat cad. No: 238, 34728, Goztepe, Istanbul, Turkey, Tel: 00905333269488; Fax 00902163636211; E-mail: zeynepozkurt@hotmail.com

Received February 21, 2014; Accepted March 26, 2014; Published March 28, 2014

Citation: Yazıcıoğlu Y, Özkurt-Kayahan Z, Özçakır-Tomruk C, Katipoğlu B (2014) Prosthetic Management of a Maxillary Alveolar Defect with an ImplantRetained Fixed Prosthesis: A Clinical Report. Dentistry 4: 225. doi:10.4172/2161 1122.1000225

Copyright: (c) 2014 Yazıcıoğlu Y, et al. This is an open-access article distributed under the terms of the Creative Commons Attribution License, which permits unrestricted use, distribution, and reproduction in any medium, provided the original author and source are credited. 


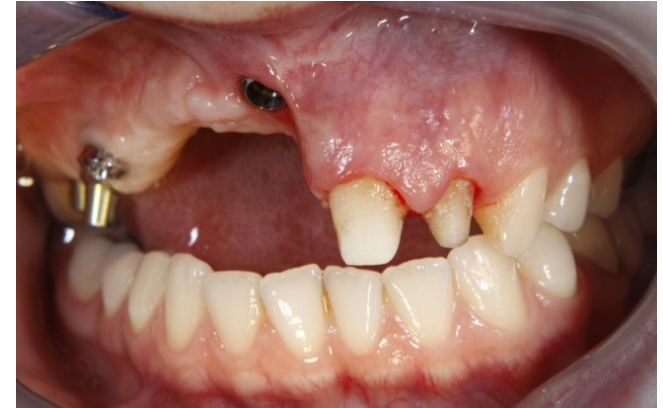

Figure 3: Implant positions before the prosthodontic procedures.

denture; therefore, an implant-supported fixed partial denture was suggested to the patient in order to restore the defect area. After the medical and dental history taking, an initial periodontal treatment was performed before any surgical and restorative procedures.

\section{Surgical procedure}

According to clinical and radiographic examination, it was clearly observed that the height of the alveolar crest was inadequate for implant placement. Sinus floor elevation was suggested to prepare the implant site. A staged protocol was planned, involving a delay of 6 months from sinus floor elevation to implant surgery.

Local infiltrative anesthesia with Articain 4\% solution with an epinephrine addition of 1:200,000 (Ultracain DS 3\%, Aventis, Bad Soden, Germany), was applied before the surgery. Following a crestal incision and vertical releasing incisions, a mucoperiosteal flap was elevated and reflected to expose the lateral wall of the sinus. The size of the lateral window was determined by the number of implants to be placed. This window was outlined with a round bur. The bony window was completely removed with the diamond bur. The Schneiderian membrane was carefully reflected to the desired extent mesially, medially, and distally. Care was taken to mobilize the Schneiderian membrane from the inner bone surface without perforation. A composite graft consisting autogenous bone chips combined approximately 1:1 with deproteinized bovine bone mineral (DBBM; Bio-Oss, Geistlich Pharma AG, Wolhusen, Switzerland) was utilized. The autogenous bone chips were harvested locally within the same flap extension. The composite graft was mixed with fresh blood from the wound, and then applied into the created space following elevation of the mucous membrane. The grafted area was then covered with a bioresorbable porcine collagen membrane consisting of collagen types I and III (Bio-Gide, Geistlich Pharma AG). Tension free flap closure was accomplished with interrupted sutures. Postoperatively, the patient was prescribed amoxicillin $1 \mathrm{~g}, 2$ times per day for 7 days, and naproxen sodium 550 mg, 2 times per day, as needed for pain. In addition, the patient was instructed to rinse with $0.12 \%$ chlorhexidine gluconate solution 3 times a day for 1 week.

After a healing period of 6 months, 4 implants (Straumann Dental Implantn System) were placed using a standardized surgical procedure, with the border of the SLA surface approximating the alveolar bone crest, leaving the machined neck portion in the transmucosal area. The posterior 3 implants $(4.1 \mathrm{~mm}$ in diameter, $10 \mathrm{~mm}$ in length) were inserted in the second premolar, first molar and second molar teeth region. A horizontal ridge augmentation was performed during implant insertion in the maxillary right central incisor region using a composite graft and the implant (3.1 $\mathrm{mm}$ in diameter, $10 \mathrm{~mm}$ in length) was placed palatally due to the defect. After the implant insertion, the mucoperiosteal flaps were repositioned and sutured in place. Similar postoperative care was given and a panoramic radiograph was taken to examine the implant positions (Figure 3). At clinical follow-up of 7 days later, a clinically healthy mucosa was observed. The patient did not complain of postoperative pain or swelling.

After a healing period of 6 months, the implants were exposed and healing abutments were connected. Two weeks later, the patient was ready for definitive acrylic resin fixed restoration. Intraoral examination revealed that the soft tissues in the region of the implants had healed well, and radiographic examination showed that the grafted bone appeared to be well integrated both with the maxillary bone and the implants.

\section{Restorative procedure}

The metal-ceramic crowns on the maxillary left central and lateral incisors were renewed. The metal-ceramic fixed partial denture was constructed between maxillary right central implant and maxillary right second molar implant for the replacement of the missing teeth according to routine prosthetic protocol. The prosthesis was also constructed with gingival-colored porcelain to compensate the hard and soft tissue loss and to enhance the lip support. The prosthesis was then inserted intraorally, the occlusion was checked to obtain a group function, and the prosthesis was screwed in its place at the manufacturer-recommended force of $35 \mathrm{Ncm}$. The access holes of the screws were sealed with gingival-colored composite resin material (Micerium HRI, Avegno, Italy). The patient was given oral hygiene instructionsincluding dental floss and interproximal brushes designed for fixed partial denture. The results were satisfactory both for the patient and for the clinician (Figures 4 and 5).

\section{Discussion}

There are various treatment options for the patients who have severe maxillary dentoalveolar defects. Although removable partial dentures are indicated in soft and hard tissue lost in order to acquire lip support [10], they are not the first choice of the patients who have high esthetic and functional expectations [6]. Conventional tooth-supported fixed partial dentures, in which the adjacent teeth have been prepared for use as abutments, is the other option for replacing missing teeth [8]. The primary reasons for suggesting fixed partial dentures are its clinical ease and reduced treatment time and costs [11]. Nowadays,

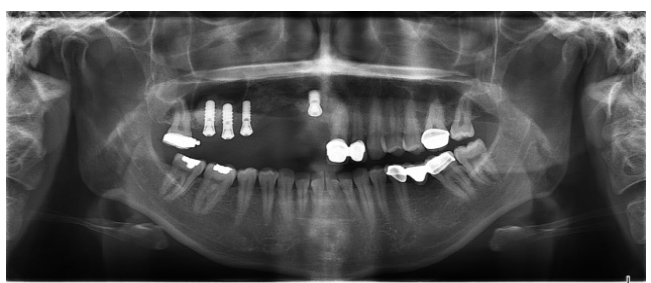

Figure 4: Panoramic radiograph showing implants.

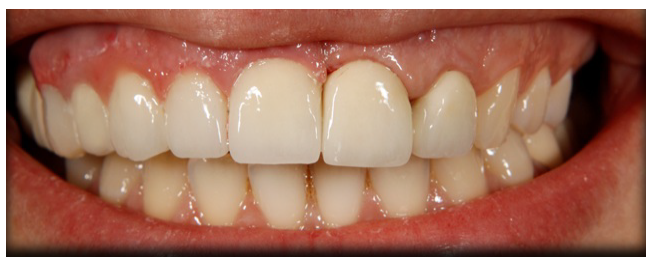

Figure 5: Intraoral view of final restoration 
Citation: Yazıcıoğlu Y, Özkurt-Kayahan Z, Özçakır-Tomruk C, Katipoğlu B (2014) Prosthetic Management of a Maxillary Alveolar Defect with an Implant-Retained Fixed Prosthesis: A Clinical Report. Dentistry 4: 225. doi:10.4172/2161-1122.1000225

dental implant treatment has become a frequent restorative procedure, offering improved esthetics and biomechanical benefits [12]. However, many patients do not want to wait a long time to complete the implant treatment [13]. They may also be reluctant to undergo a surgical intervention [14].

In this report, the patient had been used a removable partial denture for a long time, and complained about its esthetic and comfort. She was fully informed about different treatment options, refused a removable denture option, and the fabrication of implant supported fixed partial denture was considered to be most acceptable treatment option. Metal-based ceramic crowns (7-unit) were constructed, because the young female patient had high esthetic expectations. Gingivalcolored porcelain was also applied to metal-based ceramic crowns to compensate soft tissues on the maxilla. This prosthetic design offers an opportunity to support soft tissues and lip, conform to the underlying hard tissues and enhance esthetic appearance. The patient was much cooperated about to provide sufficient oral hygiene and to clear away the microbial dental plaque on the defect surface of the prosthesis.

At the 6-month recall visit, the periodontal tissues were healthy and gingival problems were not observed in the patient, because the importance of oral hygiene procedures on the maintenance and survival of the denture was emphasized.

\section{Conclusion}

This clinical report described the rehabilitation of maxillary anterior teeth and surrounding alveolar bone loss with dental implants. A metal-ceramic fixed partial denture was constructed and the use of gingival-colored porcelain enhanced the esthetic outcome. Despite the limitations of the maxillary alveolar crest, the esthetic and functional demands of the patient were fulfilled by this prosthetic rehabilitation.

\section{References}

1. Freitas $R$, Kaizer $O B$, Hamata $M M$, de Resende $D R$, de Oliveira Fortes Kaizer $R$ (2006) Prosthetic rehabilitation of a bone defect with a teeth-implant supported, removable partial denture. Implant Dent 15: 241-247.

2. Balshi TJ (1991) Oral prosthodontic rehabilitation for traumatic sports injuries Dent Clin North Am 35: 771-796.

3. Ustün Y, Esen E, Toroğlu MS, Akova T (2004) Multidisciplinary approach for the rehabilitation of dentoalveolar trauma. Dent Traumatol 20: 293-299.

4. Evren BA, Basa S, Ozkan Y, Tanyeri H, Ozkan YK (2006) Prosthodontic rehabilitation after traumatic tooth and bone loss: a clinical report. J Prosthet Dent 95: 22-25.

5. Ugurlu K, Sacak B, Huthut I, Karsidag S, Sakiz D, et al. (2007) Reconstructing wide palatomaxillary defects using free flaps combining bare serratus anterior muscle fascia and scapular bone. J Oral Maxillofac Surg 65: 621-629.

6. Frank RP, Brudivik JS, Leroux B, Milgrom P, Hawkins N (2000) Relationship between the standards of removable partial denture construction, clinical acceptability, and patient satisfaction. J Prosthet Dent 83: 521-527.

7. Salinas TJ, Block MS, Sadan A (2004) Fixed partial denture or single-tooth implant restoration? Statistical considerations for sequencing and treatment. J Oral Maxillofac Surg 62: 2-16.

8. Dhingra K (2012) Oral rehabilitation considerations for partially edentulous periodontal patients. J Prosthodont 21: 494-513.

9. Ancowitz S (2004) Esthetic removable partial dentures. Gen Dent 52: 453-459.

10. Gökçen-Röhlig B, Atalay B, Baca E, Ișik D, Meriç U (2009) Prosthetic rehabilitation of a patient with a mandibular defect caused by a gunshot wound. J Craniofac Surg 20: 1614-1617.

11. Misch CE (2005) Maxillary anterior single-tooth replacement. Mosby, St. Louis.

12. Wiens JP (1992) The use of osseointegrated implants in the treatment of patients with trauma. J Prosthet Dent 67: 670-678.

13. Sclar AG (2004) Strategies for management of single-tooth extraction sites in aesthetic implant therapy. J Oral Maxillofac Surg 62: 90-105.

14. Ozkurt Z, Kazazoglu E (2010) Treatment modalities for single missing teeth in a Turkish subpopulation: an implant, fixed partial denture, or no restoration. J Dent Sci 5: 183-188. 